\title{
Temperature Prediction of Aluminum Alloy Work-Pieces in Aging Furnaces Based on Improved Case-Based Reasoning
}

\author{
Qi Zhu, Ling Shen, Jianjun He*, Weihua Gui \\ School of Information Science and Engineering, Central South University, Changsha, China \\ Email: zhuqi2017@qq.com, shenling0409@163.com, *jjhe@csu.edu.cn,gwh@mail.csu.edu.cn
}

How to cite this paper: Zhu, Q., Shen, L., He, J.J. and Gui, W.H. (2017) Temperature Prediction of Aluminum Alloy Work-Pieces in Aging Furnaces Based on Improved Case-Based Reasoning. International Journal of Nonferrous Metallurgy, 6, 47-59. https://doi.org/10.4236/ijnm.2017.64004

Received: October 13, 2017

Accepted: October 28, 2017

Published: October 31, 2017

Copyright $\odot 2017$ by authors and Scientific Research Publishing Inc. This work is licensed under the Creative Commons Attribution International License (CC BY 4.0).

http://creativecommons.org/licenses/by/4.0/

\begin{abstract}
The temperature of aluminum alloy work-pieces in the aging furnace directly affects the quality of aluminum alloy products. Since the temperature of aluminum alloy work-pieces cannot be measured directly, a temperature prediction model based on improved case-based reasoning (CBR) method is established to realize the online measurement of the work-pieces temperature. More specifically, the model is constructed by an advanced case-based reasoning method in which a state transition algorithm (STA) is firstly used to optimize the weights of feature attributes. In other words, STA is utilized to find the suitable attribute weights of the CBR model that can improve the accuracy of the case retrieval process. Finally, the CBR model based on STA (STCBR) was applied to predict the temperature of aluminum alloy work-pieces in the aging furnace. The results of the experiments indicated that the developed model can realize high-accuracy prediction of work-pieces temperature and it has good application prospects in the industrial field.
\end{abstract}

\section{Keywords}

Prediction Model, Aluminum Alloy, Case-Based Reasoning, State Transition Algorithm, Aging Furnace

\section{Introduction}

The Aging Furnace (AF) is an important equipment for the thermal treatment of aluminum alloy work-pieces to enhance their comprehensive performance of anticorrosion property and mechanical properties, such as hardness and ultimate tensile strength [1]. These properties are directly influenced by the temperature of aluminum alloy work-pieces during the temperature holding period, 
which is called aging temperature. According to the production technology [2], the required control precision of work-pieces temperature is often strictly confined to a very narrow range about $\pm 1^{\circ} \mathrm{C}$. To obtain the qualified symmetrical high-strength work-pieces, accurate measurement of work-piece temperature in real time is of great importance. Direct measuring method is currently used to obtain the temperature of work-pieces by installing the thermocouples manually on the work-pieces before loading into the furnace each time. Due to the oscillations of the work-pieces and strong convection of the air inside the furnace, the thermocouples may detach from the work-pieces and even be damaged, leading to inaccurate measurement which causes unsuitable thermal treatment and even wasted work-pieces. Therefore, the establishment of a reasonable temperature prediction model of aluminum alloy work-pieces is of practical significance to realize precise control of the work-pieces temperature during the aging process. In order to predict the temperature of work-piece in furnace, Shen et al. [3] proposed a 3D multi-zone multi-phase thermal model based on a novel double-extrapolation finite element method, and achieved good results in a certain situation indeed. However, the complexity and randomness of the temperature field in the aging furnace are not yet clear which severely restricts the accuracy of the mechanism models. To deal with the problems, large amounts of data being measured and stored in the process industry were used to build predictive models based on artificial intelligence techniques, and these models are called Soft Sensors (SSs) [4]. Using SSs method, Yu and He [5] build a temperature measurement model for aging furnaces based on mixed kernel partial least squares algorithms (KPLS), and realized online estimation for the temperature of work-piece in furnace. However, the KPLS algorithm has a disadvantage of over-fitting which may decrease the accuracy of prediction. As a branch of artificial intelligence technique, Case-based reasoning (CBR) receives increasingly attentions and has a rapid development. It has a good interpretability, and over-fitting can be avoided by incremental learning. It is applicable to the fields with no accurate mathematical models, but with rich experiences and historical cases, such as Al-alloy factories and steelmaking industry. CBR can explore knowledge from the experienced cases to carry out solutions of new problems, usually applied to the field of weak theory in which the mathematical model cannot or be difficult to be established [6]. When a new operation condition is given, the CBR system can retrieve similar cases in the database of data being measured and stored in the industry to provide a possible predicted temperature of work-piece. The weight distribution determines the accuracy of case retrieval and affects the performance of CBR model. Genetic algorithm (GA) [7] is frequently utilized in weights optimization for CBR model. However, the GA has a property of premature phenomena [8] and low reliability [9], which may render weights less optimal and even unreasonable. In recent years, Zhou et al. [10] proposed a novel optimization algorithm, the state transition algorithm (STA), which has a better performance of global and local searches compared with GA. 
Thus, this paper introduces a novel weight allocation method that is based on STA to improve the traditional CBR system, and the improved CBR system is called STCBR. To test and verify the effectiveness of the developed method, STCBR is applied in temperature prediction of aluminum alloy work-pieces in AF. The experimental results show that STCBR can realize high-accuracy prediction of work-pieces temperature and has strong robustness.

\section{STCBR Algorithm}

STCBR model involves five processes: case representation, case retrieval, case reuse, case revise and case retain. Figure 1 shows an overview of the process.

\subsection{Case Representation}

Typically, a case model consists of two parts: feature attributes and solution attributes. Feature attributes are the mathematical description of problems, and solution attributes are solutions to problems. Therefore, the general case model of a source case can be represented by Case $_{i}$ as Equation (1):

$$
\text { Case }_{i}=\left\{f_{i 1}, f_{i 2}, \cdots, f_{i j}, \cdots, f_{i n}, s_{i 1}, s_{i 2}, \cdots, s_{i j}, \cdots, s_{i m}\right\}
$$

where $n$ is the number of feature attributes, $m$ is the number of feature attributes, $f_{i j}$ and $s_{i j}$ represent the value of feature attributes and solution attributes of the ith source case.

\subsection{Case Retrieval}

The purpose of case retrieval is to retrieve one or more cases with the maximum similarity to the new target case from the case base, by calculating the similarity between them. At present, the k nearest neighbor algorithm (KNN) is often used for case retrieval in the CBR system [11]. Based on KNN, there are three steps to obtain the cases with the maximum similarity to the target case from the case base.

At the first step, it is needed to calculate the local similarity $\operatorname{sim}_{i j}$ between

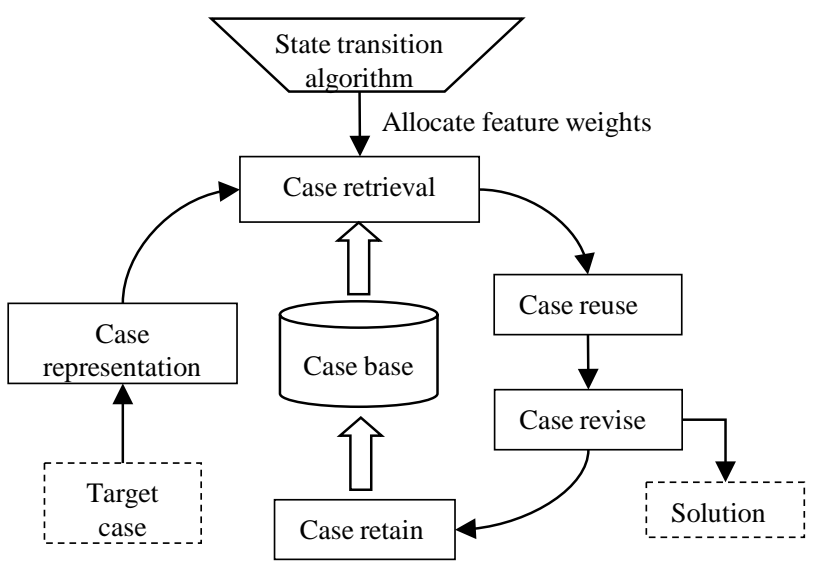

Figure 1. Flow diagram of the improved CBR. 
the target case and the source case in the case base, as is shown in Equation (2):

$$
\operatorname{sim}_{i j}=1-\frac{\left|f_{i j}-f_{j}^{\prime}\right|}{\max \left(f_{i j}, f_{j}^{\prime}\right)}
$$

where $f_{i j}$ is the value of the $t$ th feature of the $i$ th source case, and $f_{j}^{\prime}$ is the value of the $t$ th feature attribute of the target case. It is noteworthy that the local similarity describes the similarity degree of the same feature attribute between the target case and the source case. The number of feature attributes is $n$, there are thus $n$ local similarities needed to be calculated by Equation (2).

After all of the local similarities between the $i$ th source case and the target case are available, the global similarity is introduced at the second step by Equation (3):

$$
\operatorname{sim}_{i}=\sum_{j=1}^{n} w_{j} \cdot \operatorname{sim}_{i j}
$$

where $w_{j}$ is the weight of the $j$ th feature, and $n$ is the number of feature attributes.

At the third step, sort the cases by similarity based on KNN, and then the cases with maximum global similarity are chosen for case revise and reuse. The maximum global similarity is defined as Equation (4):

$$
\operatorname{sim}_{\max }=\max \left(\operatorname{sim}_{k}\right), \quad k=1,2, \cdots, \text { num }
$$

where num is the number of cases in the case base.

\subsection{Weights Allocation Based on STA}

In similarity calculation, the weights of feature attributes have a great impact on retrieved results and the accuracy of problem solving. Thus, it is important to optimize the weights of feature attributes to improve the quality of the CBR system. The optimization problem of weight allocation can be expressed as Equation (5):

$$
\left\{\begin{array}{l}
\max F=f\left(\omega_{1}, \omega_{2}, \cdots, \omega_{n}\right) \\
\text { s.t. } g(\omega)=1-\sum_{j=1}^{n} \omega_{j}=0 \\
0 \leq \omega_{j} \leq 1, j=1,2, \cdots, n
\end{array}\right.
$$

where $\boldsymbol{\omega}=\left[\omega_{1}, \omega_{2}, \cdots, \omega_{n}\right] \in R^{n}$ is the weight vector; $f(\cdot)$ is a fitness function to estimate the prediction accuracy of CBR for the training case set base on the weight vector $\boldsymbol{\omega}$. The fitness function is defined as Equation (6):

$$
f(\omega)=\sum_{l=1}^{q} \text { hit }_{l}
$$

where $q$ is the size of the training case set, and hit ${ }_{j}$ represents that if the th training case is matched successfully; if it is, hit li $_{l}$, otherwise hit h $_{l}$ is 
According to STA, a solution to an optimization problem is regarded as a state and the process of updating current solution is regarded as a state transition [12]. Speaking specifically, the weight vector $\boldsymbol{\omega}$ is considered as a state $\boldsymbol{x}$. By referring to state space representation, on the basis of current state $x(k)$, the framework of state transition algorithm can be outlined as Equation (7):

$$
\left\{\begin{array}{l}
x(k+1)=A_{k} x(k)+B_{k} u(k) \\
y(k+1)=f(x(k+1))
\end{array}\right.
$$

where $\boldsymbol{x}(k) \in R^{n}$ stands for a state corresponding to a current solution to the optimization problem; $A_{k} \in R^{n \times n}$ and $B_{k} \in R^{n \times m}$ are state transition matrices with appropriate dimensions, which are usually regarded as transformation operators for the optimization algorithm; $\boldsymbol{u}(k)$ is a function of $\boldsymbol{x}(k)$ and historical states; and $f(x(k+1))$ is the fitness function.

There are four special state transformation operators which are designed to solve the continuous optimization problems:

(1) Rotation transformation:

$$
x_{k+1}=x_{k}+\alpha \frac{1}{n\left\|x_{k}\right\|_{2}} R_{r} x_{k}
$$

where $\alpha$ is a positive constant called the rotation factor; $R_{r} \in R^{n \times n}$ is a random matrix with its entries belonging to the range of $[-1,1]$; and \|\|$_{2}$ is the 2-norm of a vector.

(2) Translation transformation:

$$
x_{k+1}=x_{k}+\beta R_{t} \frac{x_{k}-x_{k-1}}{\left\|x_{k}-x_{k-1}\right\|_{2}}
$$

where $\beta$ is a positive constant called the translation factor; $R_{t} \in R^{n \times n}$ is a random variable with its components in the range of $[0,1]$. The translation transformation will be performed only when a better solution is found.

(3) Expansion transformation:

$$
x_{k+1}=x_{k}+\gamma R_{e} x_{k}
$$

where $\gamma$ is a positive constant called the expansion factor; $R_{e} \in R^{n \times n}$ is a random diagonal matrix with its entries obeying Gaussian distribution.

(4) Axesion transformation:

$$
x_{k+1}=x_{k}+\delta R_{a} x_{k}
$$

where $\delta$ is a positive constant called the axesion factor; $R_{a} \in R^{n \times n}$ is a random diagonal matrix with its elements obeying Gaussian distribution and only one random index having a nonzero value.

The optimization flowchart of the feature weights using STA is shown in Figure 2. The detailed explanation for each phase of weights allocation based on STA is presented as follows [13]:

Phase 1 (parameters initialization). Set the values of $S E$ (the search enforce- 


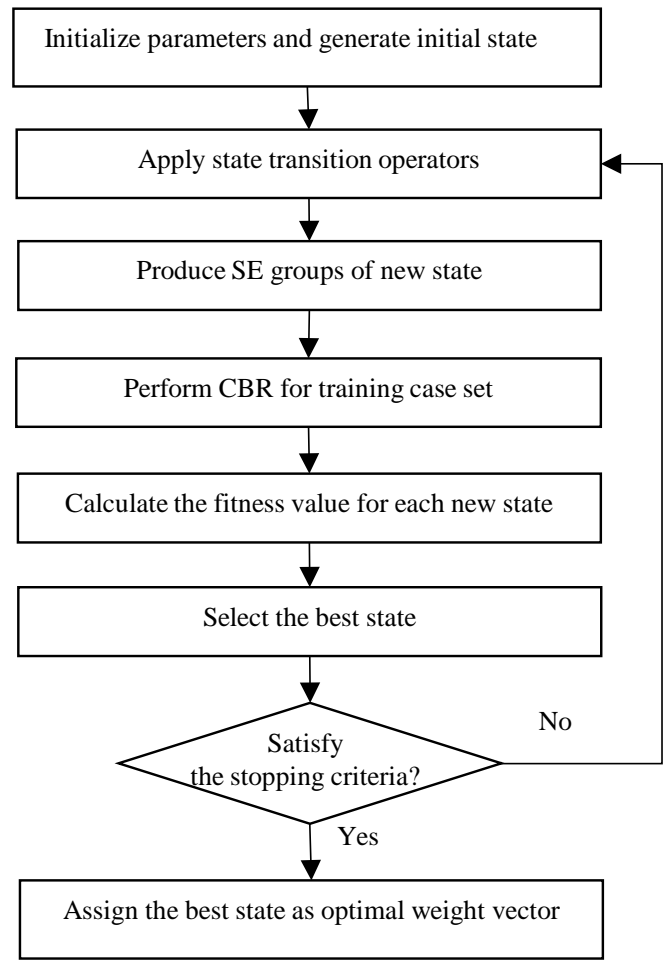

Figure 2. Flowchart of allocation of feature weights using STA.

ment, which means the times of the transformation), $\alpha, \beta, \gamma, \delta$ (operation factor), $f_{c}$ (a constant coefficient used for lessening the $\alpha$ ), and $K$ (times of iteration). Set $k=0$ and generate initial solution $x(k)$ randomly.

Phase 2 (state transition operation). Perform state transition operations for $\boldsymbol{x}(k)$. The specific operations for $\boldsymbol{x}(k)$ are given as follows:

$$
\begin{aligned}
& \text { best } \leftarrow \text { Expansion }(x(k), S E, \beta, \gamma) \\
& \text { best } \leftarrow \operatorname{Rotation}(x(k), S E, \alpha, \beta) \\
& \text { best } \leftarrow \operatorname{Axesion}(x(k), S E, \beta, \delta)
\end{aligned}
$$

Next, the operator will be described in detail, taking the expansion operator as an example:

(a) Make SE copies of $\boldsymbol{x}(k)$ and carry out an expansion operation for each copy state by Equation (10); the result is newstates $0=\left\{\boldsymbol{x}_{1}^{0}(k), \boldsymbol{x}_{2}^{0}(k), \cdots, \boldsymbol{x}_{\text {SE }}^{0}(k)\right\}$.

(b) Perform CBR process for the training data set, and then calculate the fitness value of each state of newstates by Equation (5) and make a ranking of the states according to the fitness value in descending order; and assign the state which is sorted as 1 to best.

(c) $\boldsymbol{x}(k-1) \leftarrow \boldsymbol{x}(k), \boldsymbol{x}(k) \leftarrow$ best ; make $S E$ copies of $\boldsymbol{x}(k)$ and carry out a translation operation for each copy state by Equation (9); the result is newstates $1=\left\{\boldsymbol{x}_{1}^{1}(k), \boldsymbol{x}_{2}^{1}(k), \cdots, \boldsymbol{x}_{S E}^{1}(k)\right\}$. 
(d) Perform CBR process for the training data set, and then calculate the fitness value of each state of newstates1 by Equation (5) and make a ranking of the states according to the fitness value in descending order; and assign the state which is sorted as 1 to best.

Phase 3 (iteration or termination). If $k<K$, then $k=k+1, \alpha=\alpha / f_{c}$, and go to step 2. Otherwise, assign best as the optimal weight vector $\omega^{*}$.

\subsection{Case Revise and Reuse}

Target and source cases are generally impossible to be exactly the same, thus it is significant to study how to make an appropriate adjustment for retrieved cases to achieve accurate results [14]. Based on the similar cases obtained in case retrieval process, the solution to the target case $s_{t}$ is evaluated in Equation (12):

$$
S_{t}=\frac{\sum_{i=1}^{m} \operatorname{sim}_{i} \cdot S_{i}}{\sum_{i=1}^{m} \operatorname{sim}_{i}}
$$

where $m$ is the number of cases with the maximum similarity.

\section{Experiments and Results}

To verify the validity of the improved CBR proposed in this paper, some experiments are conducted based on actual production data which were collected from an aging furnace in Southwest Aluminum Co. Ltd., China. The detailed location of the work-piece thermocouples and the working room thermocouples is shown in Figure 3. The temperature of the working room is measured by two thermocouples, A1 and A2, which are firmly installed in Area 1 and Area 2 of the working room wall. To obtain temperature information of the work-piece, two

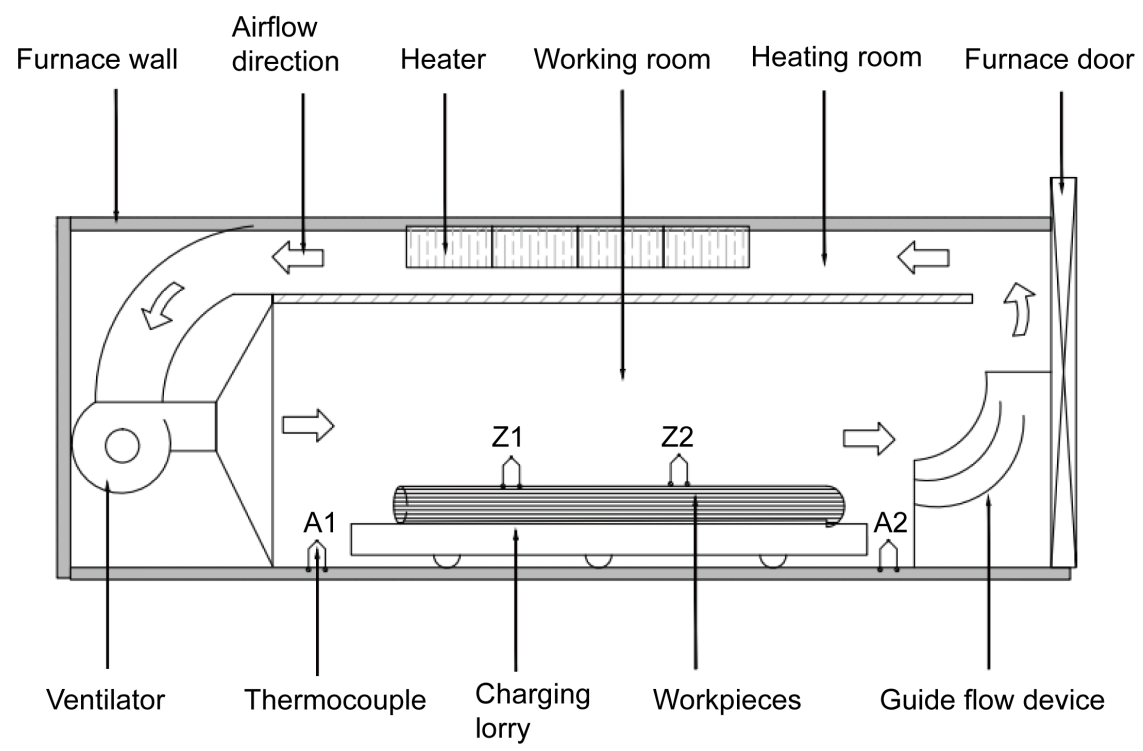

Figure 3. The structure of $12 \mathrm{t}$ aging furnace. 
thermocouples, Z1 and Z2, are installed in the Zone 1 and Zone 2 of the work-piece. Based on the production data drawn from real factory, six feature attributes are chosen from data records directly, which are working room temperature of Area $1 f_{1}$, working room temperature of Area $2 f_{2}$, the duration of heating period $f_{3}$, the duration of holding period $f_{4}$, the set temperature $f_{5}$ and alloy state $f_{6}$, respectively. Work-piece temperature of Zone $1 s_{1}$ and Work-piece temperature of Zone $2 s_{2}$ are chosen as solution attributes.

For the experiment, there are 2230 groups of data in total, and 103 groups are selected as testing data, and the remaining 2127 groups as case base and training data for feature weights allocation. The testing data comes from 2 batches of different products as shown in Table 1.

In order to validate the performance of the developed CBR model, three different CBR models were established based on the same case base. The CBR model based on STA proposed in this paper is referred to as STCBR. Furthermore, the traditional method with equal weight as EWCBR, and the genetic algorithm as GACBR:

(1) The setting parameters of STCBR [13]: the number of weight objects in the initial population is 6 , the value of search enforcement $S E$ is 30 , the times of iteration $K$ is 10 , rotation factor $\alpha$ is 1 , translation factor $\beta$ is 1 , expansion factor $\gamma$ is 1 , axesion factor $\delta$ is 1 , and the constant coefficient $f_{c}$ is 2 .

(2) In GACBR [7], the number of weight objects in the initial population is 6 , the crossover probability is 0.4 , and the mutation probability is 0.05 . Each weight object is coded using three binary numbers, and the number of iteration is 10 .

(3) EWCBR allocates average weights for each attributes.

Table 2 shows the results of feature weights allocation based on EWCBR, GACBR and STCBR, respectively. As can be seen from Table 2, all the feature weights are set equal in EWCBR model, which ignores the various influences of

Table 1. The parameters of different aluminum alloy produced in $12 \mathrm{t}$ aging furnace.

\begin{tabular}{cccccc}
\hline & \multicolumn{5}{c}{ Parameters } \\
\cline { 2 - 6 } Batch & Alloy state & $\begin{array}{c}\text { Aging temperature } \\
\text { of } 1^{\text {st }} \text { aging period } \\
\left({ }^{\circ} \mathrm{C}\right)\end{array}$ & $\begin{array}{c}\text { Holding time of } \\
1^{\text {st }} \text { aging period } \\
\text { (minute) }\end{array}$ & $\begin{array}{c}\text { Aging temperature Holding time of } \\
\text { of } 2^{\text {ed }} \text { aging period } \\
\left({ }^{\circ} \mathrm{C}\right)\end{array}$ & $\begin{array}{c}2^{\text {ed }} \text { aging period } \\
(\text { minute })\end{array}$ \\
\hline$\# 1$ & $7075-\mathrm{T} 6$ & $140 \pm 1$ & 300 & - & - \\
$\# 2$ & $7050-\mathrm{T} 6$ & $121 \pm 1$ & 210 & $177 \pm 1$ & 210 \\
\hline
\end{tabular}

Table 2. The feature weights of STCBR, GACBR and EWCBR.

\begin{tabular}{ccccccc}
\hline \multirow{2}{*}{ CBR model } & \multicolumn{5}{c}{ Weight of each feature } \\
\cline { 2 - 7 } & $f_{1}$ & $f_{2}$ & $f_{3}$ & $f_{4}$ & $f_{5}$ & $f_{6}$ \\
\hline STCBR & 0.2500 & 0.1786 & 0.2143 & 0.0357 & 0.0714 & 0.2500 \\
GACBR & 0.0833 & 0.2917 & 0.2083 & 0.1250 & 0.1667 & 0.1250 \\
EWCBR & 0.1667 & 0.1667 & 0.1667 & 0.1667 & 0.1667 & 0.1667 \\
\hline
\end{tabular}


different feature attribute on reasoning results. For GACBR and STCBR, the weights allocation methods are more reasonable and credible because they are performed based on the information of data rather than experiences of experts. For GACBR model, the weight of working room temperature of Area 2 and duration of rising period are assigned as 0.2917 and 0.2083 , which are much larger than those of the other features. It is presented that working room temperature of Area 2 and the duration of rising period play more important roles in predicting the temperature of work-pieces for GACBR model. However, for STCBR model, the alloy state and working room temperature of Area 1 are of most correlation with the temperature of work-pieces.

Figure 4 depicts the temperature profiles of different aluminum work-pieces; Figure 4(a) shows the measured temperature and predicted temperature of 7075-T6 aluminum alloy, and Figure 4(b) shows those of 7050-T6 aluminum alloy. Compared to the temperature profiles calculated by EWCBR, the temperature profiles calculated by both GACBR and STCBR have better agreement with the measured temperature curve. In order to present the prediction errors of GACBR and STCBR in a more intuitive way, Figure 5 indicates the deviation degree between the predicted temperatures and the measured temperatures. The

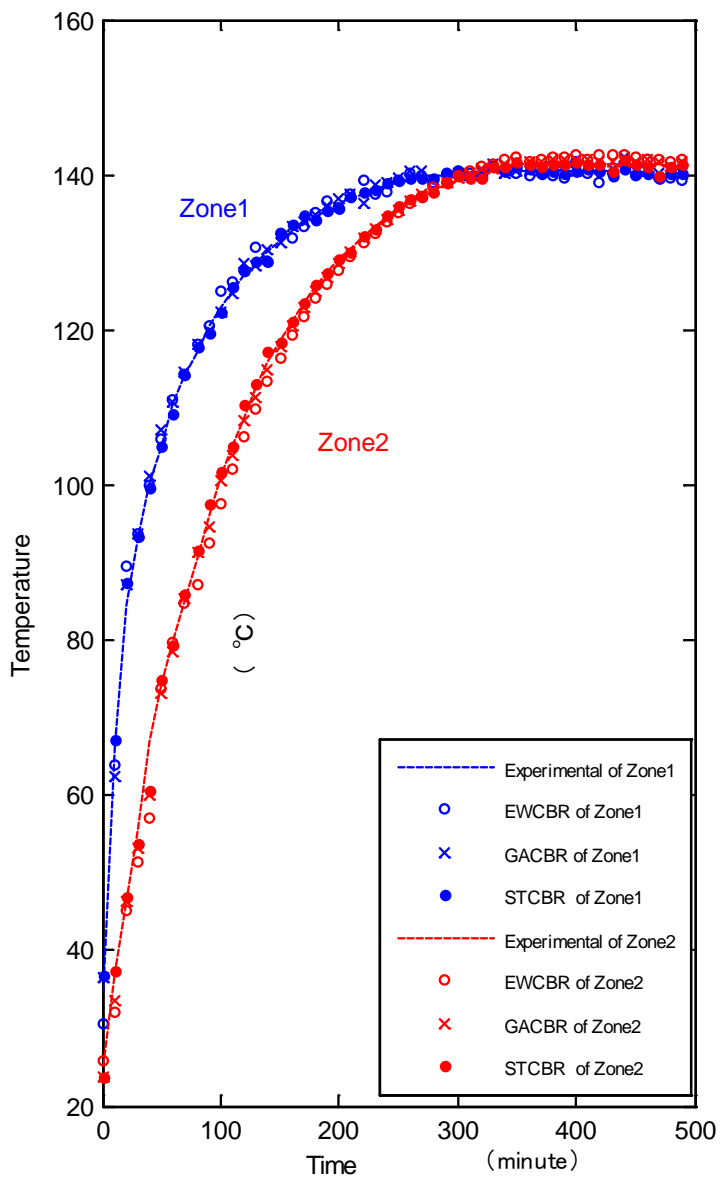

(a)

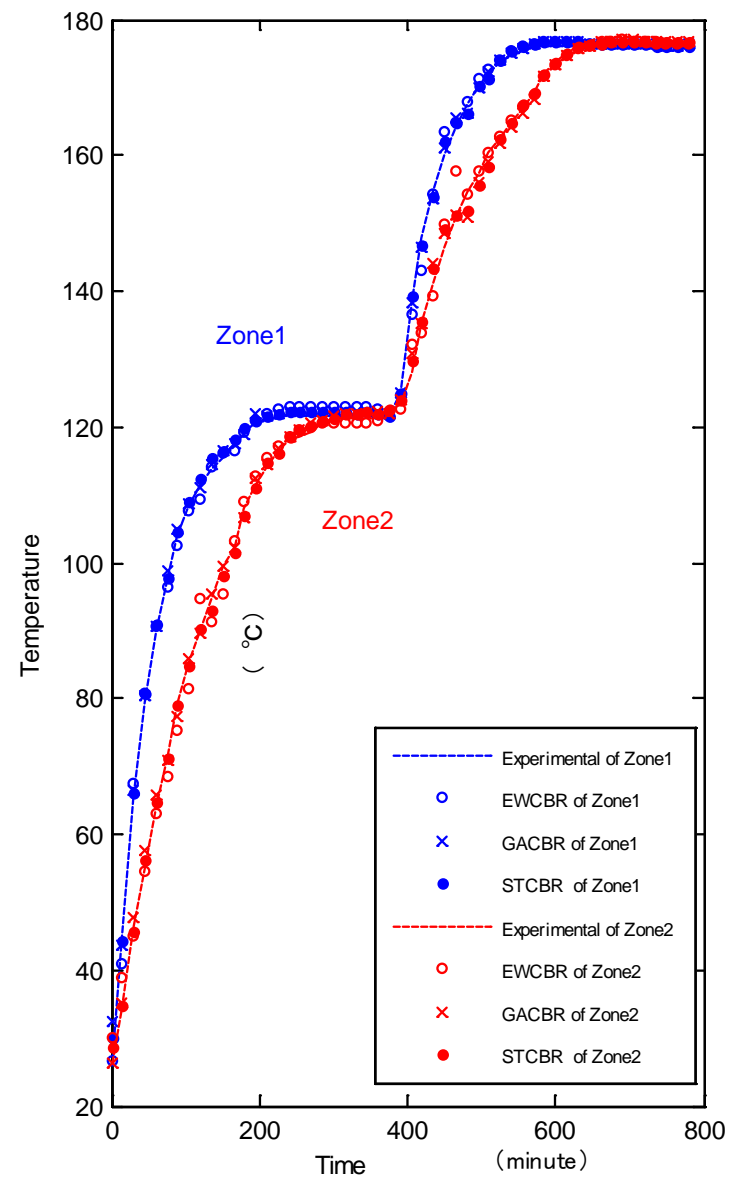

(b)

Figure 4. Prediction results of aluminum work-pieces temperature: (a) 7075-T6; (b) 7050-T6. 

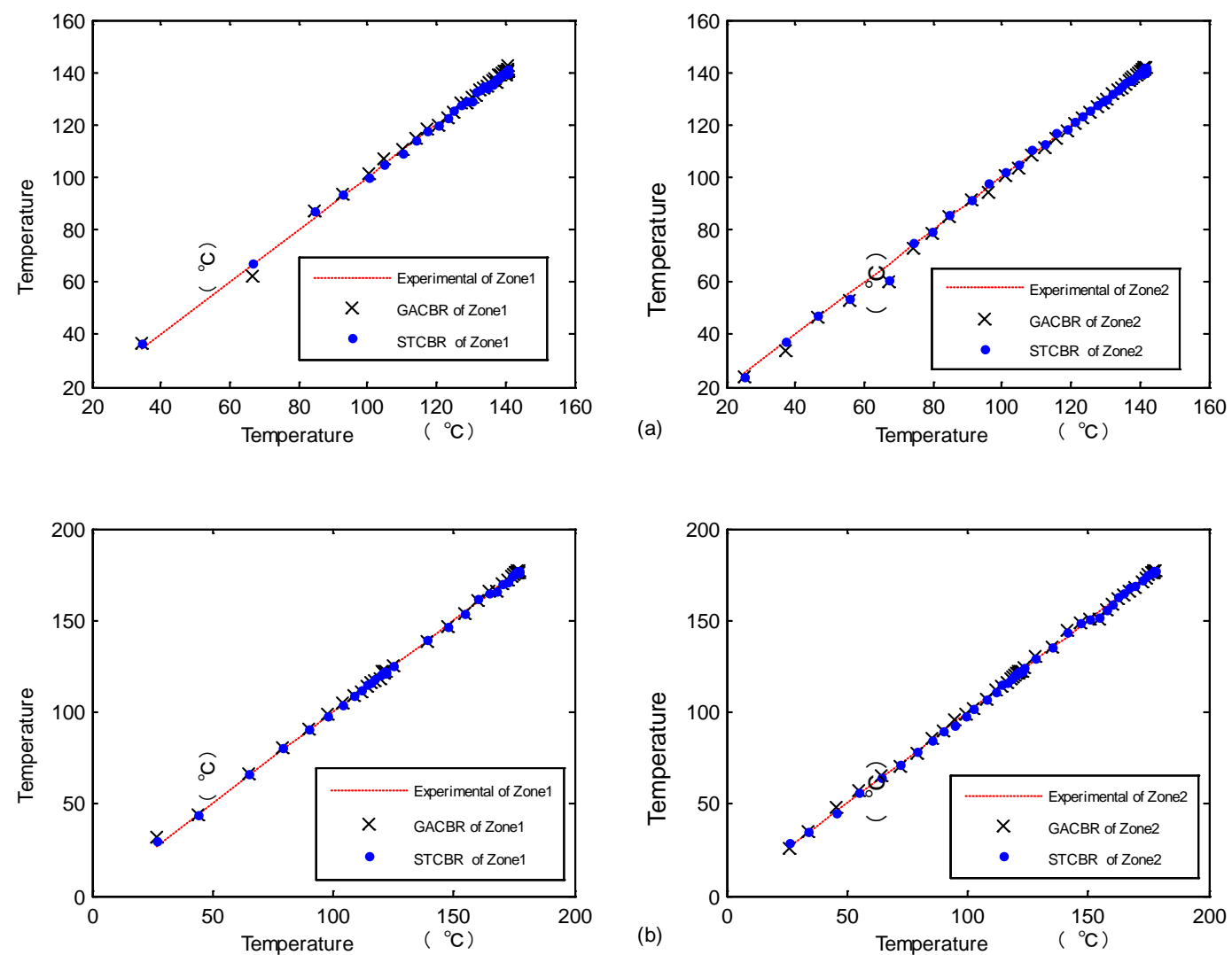

Figure 5. Comparisons of the computational accuracy of GACBR and STCBR: (a) 7075-T6; (b) 7050-T6.

closer the predicted values are to the 45 measured values, the higher the method's precision is. From Figure 5, it can be observed that, when the temperature is lower than 100, several points of the temperature calculated by GACBR have a large deviation with the measurement. In general, the prediction performance of STCBR is better than that of GACBR.

The absolute errors of different prediction models are shown in Figure 6. For both 7075-T6 and 7050-T6, in rising period, the upper and lower limits of the absolute error of STCBR are slightly better than those of GACBR and EWCBR. According to the requirement of prediction accuracy $\left( \pm 1^{\circ} \mathrm{C}\right)$ in holding period, STCBR is the only one whose prediction accuracy can meet the requirement for both 7075-T6 and 7050-T6 aluminum alloy.

To analyze the error in a numerical way, two indices Mean-SE (mean absolute error) and Max-SE (maximum absolute error) are used to evaluate the performance of each model as shown in Table 3. For the experimental results of both 7075-T6 aluminum alloy and 7050-T6 aluminum alloy, EWCBR has the largest errors in the 3 CBR models, because it ignores the various influences of different feature attribute on reasoning results which decrease the prediction accuracy of CBR system. However, the errors of GACBR and STCBR are much lower than those of EWCBR, because different feature attribute on reasoning results were considered for GACBR and STCBR. STCBR has the least mean absolute errors and least maximum absolute error. The prediction errors of STCBR may result 


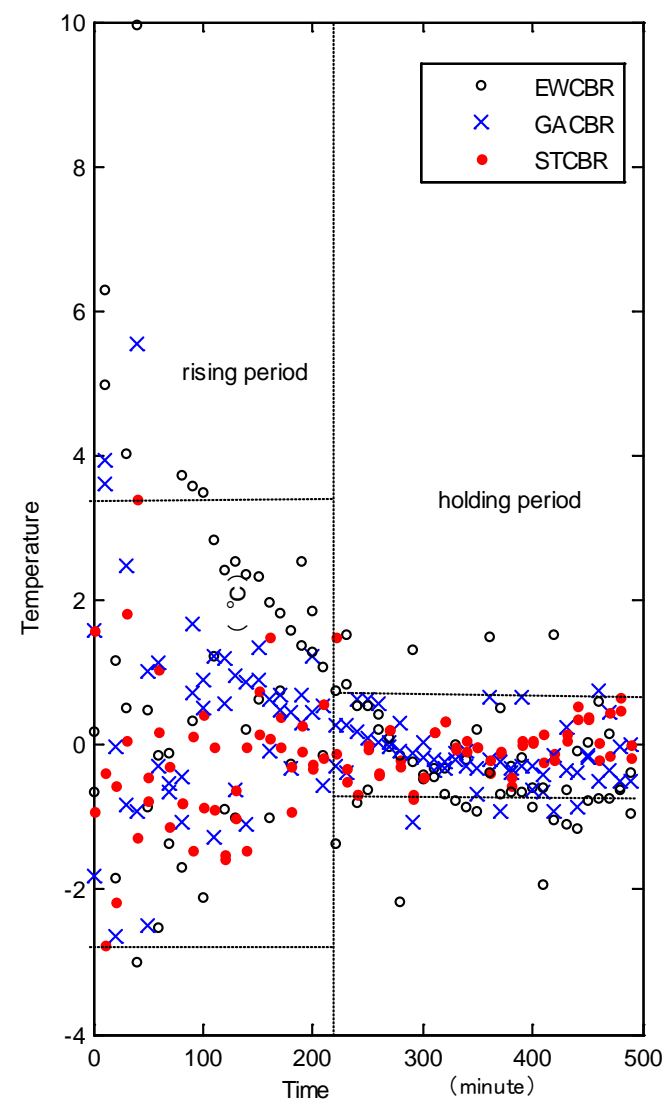

(a)

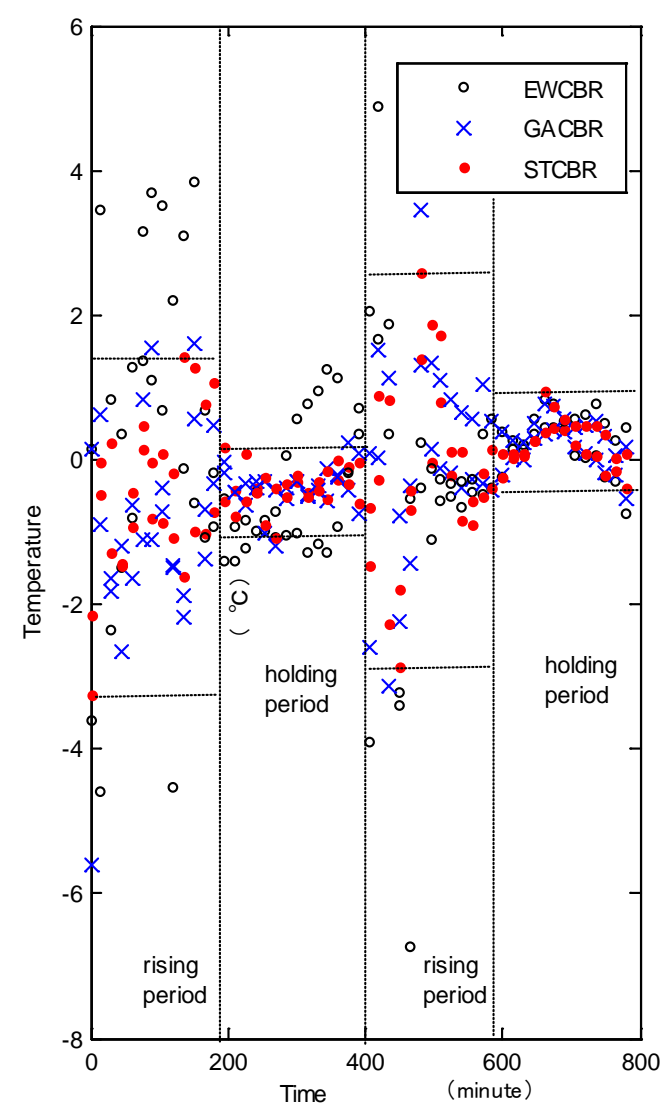

(b)

Figure 6. Prediction errors of aluminum work-pieces temperature: (a) 7075-T6; (b)7050-T6.

Table 3. Prediciton errors of different CBR models.

\begin{tabular}{ccccc}
\hline \multirow{2}{*}{ CBR model } & \multicolumn{2}{c}{ Mean absolute error $\left({ }^{\circ} \mathrm{C}\right)$} & \multicolumn{2}{c}{ Maximum absolute error $\left({ }^{\circ} \mathrm{C}\right)$} \\
\cline { 2 - 4 } & $7075-\mathrm{T} 6$ & $7050-\mathrm{T} 6$ & $7075-\mathrm{T} 6$ & $7050-\mathrm{T} 6$ \\
\hline STCBR & 0.5642 & 0.6158 & 3.2512 & 3.1438 \\
GACBR & 0.6226 & 0.6898 & 5.600 & 5.7353 \\
EWCBR & 0.9920 & 1.2873 & 9.9477 & 6.7332 \\
\hline
\end{tabular}

from disturbances of environment or insufficiency of cases.

Table 4 shows the advantages and disadvantages of these CBR models used in this paper. Obviously, the good results indicate that the prediction model based on STCBR not only has higher accuracy of prediction, but also has strong robustness when tested on different aluminum alloys.

\section{Conclusions}

Due to the significant role of online prediction of the work-pieces temperature for aging furnace, an improved CBR method was introduced in establishing the temperature prediction model. In order to promote the performance of problem solving of the CBR model, a novel optimization approach STA which combines 
Table 4. Advantages and disadvantages of different CBR models.

\begin{tabular}{|c|c|c|c|}
\hline CBR model & Advantages & Disadvantages & Application \\
\hline EWCBR & $\begin{array}{l}\text { Simple weights allocation } \\
\text { method and low } \\
\text { modeling complexity. }\end{array}$ & $\begin{array}{c}\text { Low prediction accuracy, } \\
\text { lower reliability and } \\
\text { robustness }\end{array}$ & $\begin{array}{c}\text { The system which has rich } \\
\text { expert experience and } \\
\text { mechanism knowledge }\end{array}$ \\
\hline GACBR & High prediction accuacy & $\begin{array}{l}\text { Longer time spend of } \\
\text { weights allocation, lower } \\
\text { reliability and robustness. }\end{array}$ & $\begin{array}{l}\text { The system which has certain } \\
\text { expert experience with little } \\
\text { mechanism knowledge }\end{array}$ \\
\hline STCBR & $\begin{array}{l}\text { High prediction accuacy } \\
\text { and strong robustness }\end{array}$ & $\begin{array}{l}\text { Longer time spend of } \\
\text { weights allocation }\end{array}$ & $\begin{array}{c}\text { The system which has certain } \\
\text { expert experience with little } \\
\text { mechanism knowledge }\end{array}$ \\
\hline
\end{tabular}

global search with local search to avoid the local minima is proposed to optimize feature weights. Then the established model was verified by the practical production data in AF, and the experiment results show the advantages of the STCBR model, which effectively promotes the prediction accuracy of traditional CBR in general and has practical value to apply to the aging furnace in industry.

Future research may be focused on enriching the existing STA models and improving the case revise strategy to realize better prediction performance.

\section{Acknowledgements}

This research was supported by National Natural Science Foundation of China (61174132) and Doctoral Fund of Ministry of Education of China (20130162110067). These financial contributions are gratefully acknowledged.

\section{References}

[1] Sevim, I., Hayat, F., Kaya, Y., Kahraman, N. and Sahin, S. (2013) The Study of MIG Weldability of Heat-Treated Aluminum Alloys. The International Journal of Advanced Manufacturing Technology, 66, 1825-1834.

https://doi.org/10.1007/s00170-012-4462-Z

[2] Shen, L., He, J.J., Yang, C.H. and Gui, W.H. (2016) Temperature Uniformity Control of Large-Scale Vertical Quench Furnace for Aluminum Alloy Thermal Treatment. IEEE Trans on Control Systems Technology, 24, 24-39. https://doi.org/10.1109/TCST.2015.2417495

[3] Shen, L., He, J.J., Yang, C.H. and Gui, W.H. (2017) Multi-Zone Multi-Phase Temperature Field Modelling of Aluminum Alloy Workpieces in Large-Scale Vertical Quench Furnaces. Applied Thermal Engineering, 113, 1569-1584. https://doi.org/10.1016/j.applthermaleng.2016.11.058

[4] Kadlec, P., Gabrys, B. and Strandt, S. (2009) Data-Driven Soft Sensors in the Process Industry. Computers and Chemical Engineering, 33, 795-814. https://doi.org/10.1016/j.compchemeng.2008.12.012

[5] Yu, Q. and He, J.J. (2017) Temperature Soft Sensor Method for Molded Aging Ovens. Information and Control, 3, 328-334.

[6] Guo, Y., Hu, J. and Peng, Y.H. (2011) Research on CBR System Based on Data Mining. Applied Soft Computing, 11, 5006-5014.

[7] Ahn, H. and Kim, K. (2009) Global Optimization of Case-Based Reasoning for 
Breast Cytology Diagnosis. Expert Systems with Applications, 36, 724-734.

[8] Eko, J.W., Djoko, S., Wawan, G.A.K. and Susanti, A. (2014) Designing a Genetic Algorithm for Efficient Calculation in Time-Lapse Gravity Inversion. Journal of Engineering and Technological Sciences, 46, 58-77.

https://doi.org/10.5614/j.eng.technol.sci.2014.46.1.4

[9] Yang, C.X. and Yan, X.F. (2011) A Fuzzy-Based Adaptive Genetic Algorithm and Its Case Study in Chemical Engineering. Chinese Journal of Chemical Engineering, 19, 299-307. (In Chinese)

[10] Zhou, X.J., Yang, C.H. and Gui, W.H. (2012) State Transition Algorithm. Journal of Industrial and Management Optimization, 8, 1039-1056.

https://doi.org/10.3934/jimo.2012.8.1039

[11] Yan, A.J., Shao, H.S. and Guo, Z. (2014) Weight Optimization for Case-Based Reasoning using Membrane Computing. Information Sciences, 287, 109-120.

[12] Zhou, X.J., Yang, C.H. and Gui, W.H. (2014) Nonlinear System Identification and Control using State Transition Algorithm. Applied Mathematics and Computation, 226, 169-179.

[13] Wang, Y.L., He, H.M., Zhou, X.J., Yang, C.H. and Xie, Y.F. (2016) Costs and Energy Efficiency in the Slumina Evaporation Process by A Multi-Objective State Transition Algorithm. Canadian Journal of Chemical Engineering, 94, 53-65. https://doi.org/10.1002/cjce.22353

[14] Zhu, G.N., Hu, J., Qi, J. and Peng, Y.H. (2015) An Integrated Feature Selection and Cluster Analysis Techniques for Case-Based Reasoning. Engineering Applications of Artificial Intelligence, 39, 14-22. 